\title{
Unusual microbes in asthma exacerbation: Alcaligenes xylosoxidans and Leishmania
}

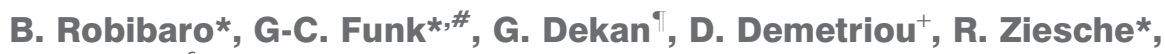 \\ S. Winkler ${ }^{\S}$ and L.H. Block*
}

ABSTRACT: Asthma is a chronic inflammatory condition characterised by a variable degree of airflow limitation. Exacerbations during the course of asthma often occur due to environmental factors or infectious, mostly viral, aetiology.

The present study reports the case of a 61-yr-old male with severe asthma hospitalised due to increasing respiratory distress. Since recovery was delayed despite anti-obstructive/antiinflammatory and antibiotic therapy, further diagnostic procedures, including bronchoscopy, were performed in order to attempt to identify the cause of the worsening respiratory condition.

The surprising finding consisted of a rare coincidence of concomitant infection with the bacterial pathogen Alcaligenes xylosoxidans, grown from bronchoalveolar lavage fluid, and the protozoan parasite Leishmania spp., revealed by histopathological examination of bronchial mucosal biopsy specimens. This is the first report of an isolated bronchial mucosal involvement of Leishmania in an HIV-negative asthma patient following brief exposure in Leishmania-endemic regions. Further, to the best of the present authors' knowledge, this represents the first description of $A$. xylosoxidans in asthma, although it is questionable whether it was an infection or colonisation.

The present observation identifies previously unreported microbial pathogens associated with asthma exacerbation. Further, the report highlights the importance of obtaining a thorough travel history and applying invasive diagnostic procedures in circumstances of treatment failure, even under unfavourable conditions.

KEYWORDS: Alcaligenes xylosoxidans, asthma, bronchoscopy, corticosteroids, exacerbation, Leishmania spp.

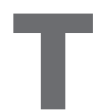
he Gram-negative water-borne bacterium Alcaligenes xylosoxidans is frequently resistant to multiple antibiotics. Reports of infection with A. xylosoxidans include bacteraemia, intravascular-catheter-associated infections, endocarditis, pneumonia, peritonitis, meningitis and infections in cystic fibrosis, in both immunocompetent and immunocompromised hosts [1].

Leishmaniasis is a vector-borne disease caused by the protozoan pathogen Leishmania, and transmitted to the human host through the bite of sandflies. Disease severity is mainly determined by the species of transmitted parasite and the capacity of the host immune response, and ranges from a subclinical course to generalised systemic disease with fatal outcome. Most reports on leishmaniasis comprise immunosuppressed people, in particular patients with HIV or iatrogenic immunosuppression, mainly following solid organ or haematopoietic stem cell transplantation $[2,3]$.

\section{CASE REPORT}

A 61-yr-old male with cortisone-dependent bron-

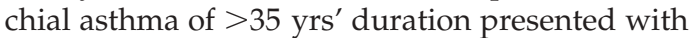
progressing dyspnoea and phlegm. On admission, the patient complained of dyspnoea and coughing and was expectorating purulent sputum. He had no fever, was an Austrian-born citizen and never-smoker, and had no pets. He was taking oral triamcinolone, amlodipine, lansoprazole, inhaled salmeterol/fluticasone and tiotropium. The travel history of the retired architect included visits to Nepal and Thailand 5 yrs earlier; in the last $2 \mathrm{yrs}$, he had been to Tunisia, Turkey and, $\sim 3$ weeks prior to admission, Portugal.

\section{AFFILIATIONS}

Depts of *Internal Medicine II, Division of Pulmonary Medicine, ${ }^{+}$Internal Medicine III, Division of Nephrology, and

§Internal Medicine I, Division of Infectious Diseases and Tropical Medicine, and

"Clinical Institute of Pathology, Vienna General Hospital and Medical University of Vienna, and \#Dept of Respiratory and Critical Care MedicineOtto Wagner Hospital Vienna, Austria.

CORRESPONDENCE

B. Robibaro

Medical University Vienna, Dept of Internal Medicine II,

Division of Pulmonary Medicine

General Hospital Vienna

Waehringer Guertel 18-20

A-1090 Vienna

Austria

Fax: 431404004784

E-mail: bruno.robibaro@

meduniwien.ac.at

Received:

August 112008

Accepted after revision:

November 212008

STATEMENT OF INTEREST

None declared. 
His respiratory situation showed severe obstruction, with a forced expiratory volume in one second of $1.1 \mathrm{~L}(29 \%$ of the predicted value), and severe hypoxaemia, with an arterial oxygen tension $\left(\mathrm{Pa}_{\mathrm{O}} \mathrm{O}_{2}\right)$ of $5.9 \mathrm{kPa}(44 \mathrm{mmHg})$. The haemoglobin concentration was $12.6 \mathrm{mg} \cdot \mathrm{dL}^{-1}$, and the total leukocyte count $11.9 \times 10^{6}$ cells $\cdot \mathrm{L}^{-1}$. No eosinophilia was detected on differential blood cell count and the immunoglobulin E level was within the normal range. C-reactive protein levels were normal and the erythrocyte sedimentation rate was slightly elevated (26 mm in first hour and $32 \mathrm{~mm}$ in second). Electrophoresis showed mild hypogammaglobulinaemia $\left(5.5 \mathrm{~g} \cdot \mathrm{L}^{-1}\right)$. Testing for HIV gave negative results. Initial empirical antimicrobial treatment with moxifloxacin was started and intensified anti-obstructive/anti-inflammatory therapy, including systemic corticosteroids, was given.

Since recovery was delayed despite therapy, the patient underwent computed tomography (CT) of the chest, which revealed signs of bronchitis/bronchiolitis with intraluminal retention of mucus and small bilateral pleural effusions without pulmonary infiltrates. In addition, fibreoptic bronchoscopy was carried out. Microbiological analysis of bronchoalveolar lavage (BAL) fluid revealed growth of $A$. xylosoxidans at a concentration of $1 \times 10^{4}$ cells $\cdot \mathrm{mL}^{-1}$. Since particularly severely inflamed and swollen bronchial mucosa with a tumour-like aspect was seen in the lower trachea and main bronchial tree (fig. 1), endobronchial biopsy specimens of the mucosa were taken. Histopathological examination of the biopsy specimens showed extensive inflammation with abundant intracellular Leishmania amastigotes (fig. 2). In the BAL fluid, only few macrophages with intracellular inclusions consistent with Leishmania parasites were seen (fig. 3). The liver and spleen were not enlarged, which was confirmed by CT. Serum testing for antibodies directed against $L$. donovani by

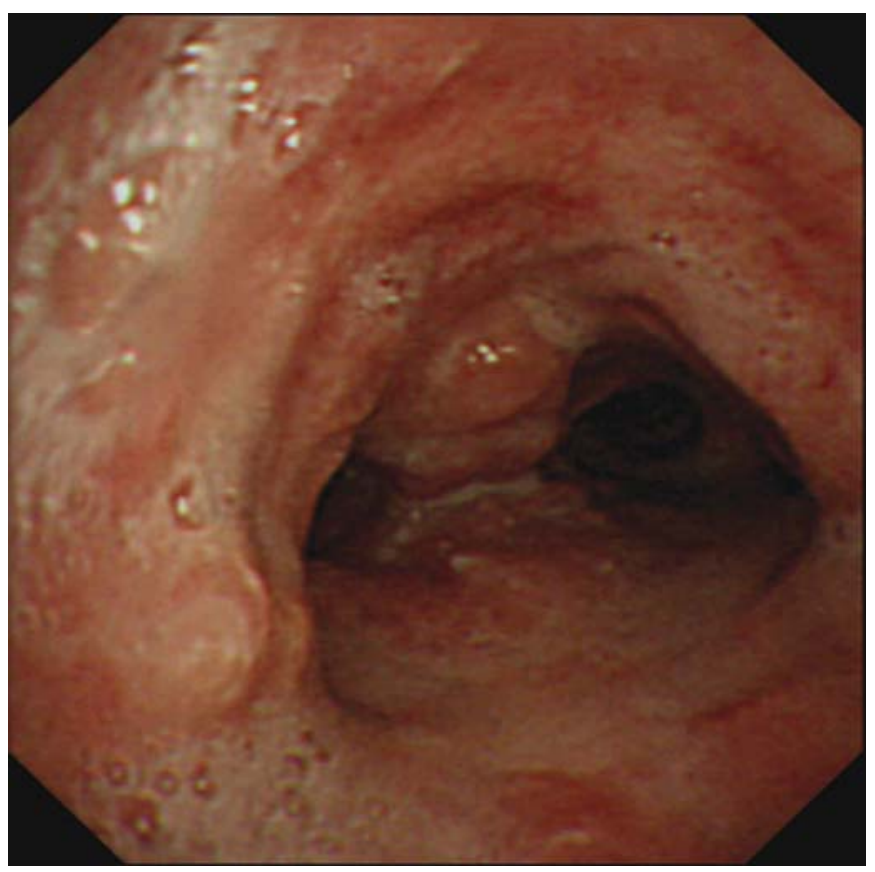

FIGURE 1. Bronchoscopic image of the distal third of the trachea showing severely inflamed endobronchial mucosa with a tumour-like aspect. indirect immunofluorescence test was negative, as was that by ELISA. In addition, a bone marrow aspirate was negative for parasite detection.

Antileishmanial chemotherapy was started with $3 \mathrm{mg} \cdot \mathrm{kg}$ body weight $^{-1}$ liposomal amphotericin B on days $1-5,10$ and 14 . The patient improved along with showing better pulmonary gas exchange, with a $\mathrm{Pa}_{1} \mathrm{O}_{2}$ of up to $9.3 \mathrm{kPa}(70 \mathrm{mmHg})$, and was eventually discharged. He refused further control visits, as well as a recommended re-examination, and was lost to follow-up.

\section{DISCUSSION}

The present report, at least to the best of the present authors' knowledge, represents the first case of bronchial asthma exacerbation with recovery of $A$. xylosoxidans from BAL fluid, as well as the first report of bronchial mucosal leishmaniasis in an HIV-negative patient following brief exposure in Leishmania-endemic regions.

A. xylosoxidans is a Gram-negative rod known to colonise and cause exacerbations in the airways of patients with cystic fibrosis [1, 4]. Interestingly, despite several reports of $A$. xylosoxidans infection in cystic fibrosis, no cases of $A$. xylosoxidans infection in other chronic respiratory diseases, including bronchial asthma, have been reported to date. Although, in the literature, A. xylosoxidans is most often described as a resistant microorganism, including resistance to aminoglycosides and quinolones, in the present case, the strain was sensitive to quinolones.

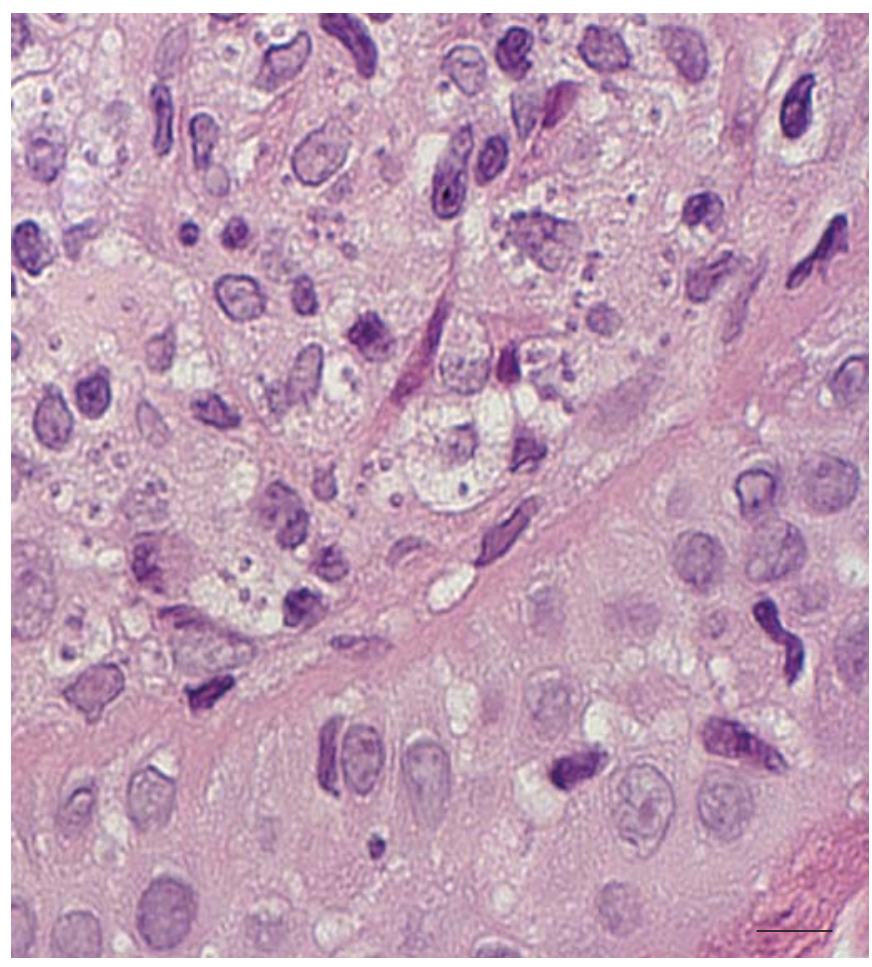

FIGURE 2. Histology of endobronchial mucosal tissue taken from the patient's lower trachea before treatment. Photomicrograph of endobronchial biopsy specimen using high-power magnification (haematoxylin-eosin stain); several intracellular engulfed Leishmania spp. can be identified. Scale bar $=14 \mu \mathrm{m}$. 


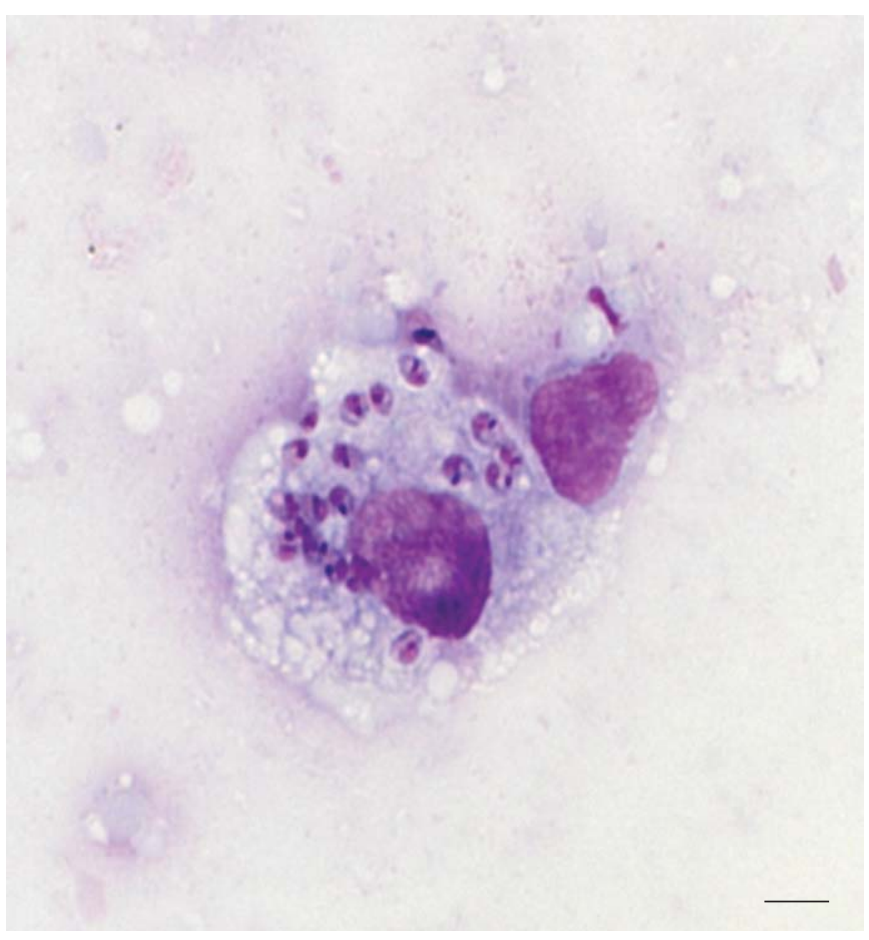

FIGURE 3. Intracellular Leishmania spp. in bronchoalveolar lavage fluid macrophage (Giemsa stain). Scale bar $=6 \mu \mathrm{m}$.

Leishmaniasis remains a frequent disease around the globe, accounting for $\sim 200,000$ new cases annually and $\sim 70,000$ deaths $\cdot \mathrm{yr}^{-1}$, and affecting mostly people in lowincome countries. However, people from western countries are increasingly encountering the disease for reasons of augmented travelling [5]. In times of globalisation, people immigrating from endemic regions may also potentially be infected, which highlights the need for disease awareness even in non-endemic regions. Emergence of the HIV/AIDS pandemic and the widespread use of immunosuppressive drugs, including long-term systemic corticosteroids for various reasons, are factors associated with an increased risk of contracting leishmaniasis, provided that an exposure in endemic areas has occurred [2,3,6].

Both of the aforementioned prerequisites were met in the present case. The patient was on frequent systemic and longterm inhaled corticosteroids due to his chronic respiratory illness and had repeatedly travelled into Leishmania-endemic regions. Although a common bacterial exacerbation of asthma was assumed, bronchoscopy revealed another unexpected pathogen. Intracellular Leishmania amastigotes in alveolar macrophages could be recovered from BAL fluid, as well as Leishmania amastigotes in biopsy specimens from the bronchial mucosa. If bronchoscopy had not been performed, the diagnosis would have been missed, perhaps with deleterious consequences for the patient. Consecutive therapy with amphotericin B may have brought a definitive parasitic eradication, which could not be proven since the patient was not seen again following discharge from hospital.

Several cases of pulmonary leishmaniasis have been reported, most of them in HIV-co-infected patients or patients who had undergone transplantation [2, 3]. In addition, mucosal and mucocutaneous leishmaniasis involving the oral or nasal mucosa, larynx or pharynx have been reported $[5,7,8]$, although involvement of the deeper airways was never reported in any of these cases. Another report was of a case of leishmaniasis of the trachea with consequent tracheal stenosis in a French patient [9]. A systematic post mortem study demonstrated interstitial pneumonitis in 10 (77\%) out of 13 cases as part of a general infection [10]. However, amastigotes within macrophages were identified in only three cases. Concerning bronchial involvement due to leishmaniasis, only one case has been reported to date. In this report, the patient underwent bronchoscopy following unsuccessful treatment of persisting hilar and mediastinal lymphadenopathy. Akin to the present case, bronchoscopy showed severe inflammatory changes throughout the bronchial tree and lower trachea. However, histological examination of bronchial biopsy specimens from these lesions revealed only noncaseating granulomata, and parasites could not be detected. Instead, leishmaniasis was diagnosed through detection of L. donovani DNA by PCR. In this case, the infection had occurred in an immunocompetent patient originally from an endemic area [11], whereas the present patient was a traveller with only brief documented exposure in endemic areas.

Why the present patient developed leishmaniasis of the bronchial mucosa remains elusive. As previously mentioned, corticosteroid-taking subjects have developed leishmaniasis, although pulmonary involvement was not described [6]. In patients treated with long-term corticosteroids, disseminated mucocutaneous leishmaniasis [12], fatal visceral leishmaniasis [13], cutaneous and visceral leishmaniasis in a patient with systemic sarcoidosis [14], isolated lingual leishmaniasis as well as combined cutaneous and visceral leishmaniasis [6], and laryngeal leishmaniasis [15] have been reported since the early 2000s. Corticosteroids are known to negatively interfere with antigen processing and elimination of intracellular pathogens. An adequate immune response towards intracellular infection with Leishmania amastigotes requires the mounting of an effective T-helper cell (Th) type 1 immune response, whereas the development of a Th2 profile alleviates infection and persisting inflammation. It can only be speculated that the long-standing both systemic and inhaled immunosuppressive glucocorticoid therapy may have contributed to the susceptibility of the bronchial mucosa as a disease target through impairment of the local immune defence. The presence of a significant number of usually opportunistic bacteria, such as $A$. xylosoxidans, points to a weak bronchial defence system, whether this was a sign of infection or merely increased colonisation.

Travelling abroad has become a common habit, even for those with chronic disease. The present case suggests a link between exacerbation of asthma, A. xylosoxidans and Leishmania infection, whereby the intense bronchial inflammation caused by the protozoan was capable of further destabilising the chronic inflammatory condition in the airways. Asthma is a typical example of a disease characterised by a Th2-polarised adaptive immune response. In addition, glucocorticoids favour Th2-dominated immunity and directly interfere with Th1 signalling [16]. Effective clearance of Leishmania, however, requires a distinct Th1-dominated immune response. It is thus 
conceivable that, in the present case, both the previous application of glucocorticoids and the increase in glucocorticoids during infection may have counteracted an effective removal of the protozoan. In addition, immunodepression seems to predispose towards development of mucosal leishmaniasis caused by viscerotropic strains [3].

In conclusion, in chronic respiratory diseases, microbiological analyses may reveal uncommon opportunistic pathogens such as $A$. xylosoxidans. In addition, during long-standing immunosuppressive treatment, Leishmania spp. should be considered as a differential diagnosis of exacerbation in people with a travel history in endemic regions. Moreover, this case stresses the need to apply invasive diagnostic strategies once routine diagnostics and therapy have failed, even under unfavourable conditions, such as severe respiratory insufficiency.

\section{REFERENCES}

1 Duggan JM, Goldstein SJ, Chenoweth CE, Kauffman CA, Bradley SF. Achromobacter xylosoxidans bacteremia: report of four cases and review of the literature. Clin Infect Dis 1996; 23: 569-576.

2 Pintado V, Martín-Rabadán P, Rivera Ml, Moreno S, Bouza E, Visceral leishmaniasis in human immunodeficiency virus (HIV)-infected and non-HIV-infected patients. Medicine 2001; 80: 54-73.

3 Antinori S, Cascio A, Parravicini C, Bianchi R, Corbellino M. Leishmaniasis among organ transplant recipients. Lancet Infect Dis 2008; 8: 191-199.

4 Davies JC, Rubin BK. Emerging and unusual Gramnegative infections in cystic fibrosis. Semin Respir Crit Care Med 2007; 28: 312-321.

5 Ahluwalia S, Lawn SD, Kanagalingam J, Grant H, Lockwood DNJ. Mucocutaneous leishmaniasis: an imported infection among travellers to central and South America. BMJ 2004; 329: 842-844.
6 Pittalis S, Nicastri E, Spinazzola F, et al. Leishmania infantum leishmaniasis in corticosteroid-treated patients. BMC Infect Dis 2006; 6: 177.

7 Grant A, Spraggs PD, Grant HR, Bryceson AD. Laryngeal leishmaniasis. J Laryngol Otol 1994; 108: 1086-1088.

8 Aliaga L, Cobo F, Mediavilla JD, et al. Localized mucosal leishmaniasis due to Leishmania (Leishmania) infantum: clinical and microbiologic findings in 31 patients. Medicine 2003; 82: 147-158.

9 Piens MA, Arpin D, Rabodonirina M, Loire R, Brune J. Tracheal stenosis of inflammatory aspect. Rev Pneumol Clin 1997; 53: 150-152.

10 Duarte MI, da Matta VL, Corbett CE, Laurenti MD, Chebabo R, Goto H. Interstitial pneumonitis in human visceral leishmaniasis. Trans $R$ Soc Trop Med Hyg 1989; 83: 73-76.

11 Marshall BG, Kropf P, Murray K, et al. Bronchopulmonary and mediastinal leishmaniasis: an unusual clinical presentation of Leishmania donovani infection. Clin Infect Dis 2000; 30: 764-769.

12 Motta ACF, Arruda D, Souza CS, Foss NT. Disseminated mucocutaneous leishmaniasis resulting from chronic use of corticosteroid. Int J Dermatol 2003; 42: 703-706.

13 Garcia-Cordoba F, Ortuño FJ, Segovia M, Gozalez Diaz G. Fatal visceral leishmaniasis, with massive bone-marrow infection, in an immunosuppressed but HIV-negative Spanish patient, after the initiation of treatment with meglumine antimoniate. Ann Trop Med Parasitol 2005; 99: 125-130.

14 Chianura L, Cirasino L. Leishmania infection in a 51-yearold woman with sarcoidosis. APMIS 2006; 114: 825-827.

15 Fsadni C, Fsadni P, Piscopo T, Mallia Azzopardi C. Laryngeal leishmaniasis in Malta. J Infection 2007; 54: e61-e63.

16 Liberman AC, Refojo D, Druker J, et al. The activated glucocorticoid receptor inhibits the transcription factor $\mathrm{T}$ bet by direct protein-protein interaction. FASEB J 2007; 21: 1177-1188. 